\title{
A dynamic lighting system for workplaces deficient of daylight
}

\author{
T. Koppel \& P. Tint \\ Tallinn University of Technology, Estonia
}

\begin{abstract}
This study introduces a new dynamic lighting system exceptional from typical indoor lighting solutions. The main highlights of the tested system are the variable colour temperature and the vertical lighting. Typical modern office lighting is gas tube fluorescent lighting with a fixed colour temperature. The authors used a previously developed dynamic lighting model and tested an experimental lighting panel at workplaces deficient of daylight. The selected building was an atrium type with workrooms facing the inner courtyard being the focal point of this study. The authors presumed workers in such office places to show most improvement under the intervention period. Twelve people participated in the experiment. The subjects' workplaces were improved with experimental dynamic lighting set-ups and their experience recorded using a questionnaire and a computerized reaction speed test. By the end of the experiment half of the subjects reported having benefited at least to some degree from the dynamic lighting panel. Those people reported having an improved general well-being as well feeling more productive. Analysis of reaction-speed tests also showed some improvement.
\end{abstract}

Keywords: dynamic lighting, natural daylight, colour temperature, atrium.

\section{Introduction}

The atrium-type buildings (fig. 1) are used both in cold and warm climate countries [2, 25], mainly as commercial centres, but also as educational buildings. This study focuses on the lighting conditions in the later one. In a typical workroom facing the atrium side (fig. 2), the artificial lighting is the main light source since the natural daylight alone does not come close to the required illuminance levels (500 lux for the office workplaces). 
People prefer to have windows in their workrooms. Boyce et al. [2] established that workers in offices without windows spend a small but statistically significantly more time talking to others, either face to face or by telephone; and a small but statistically significantly less time working on their computer, as compared to workers in windowed offices. An office desk situated near a window typically receives five times as much light from daylight as it would from electric lighting alone. Workers in offices without windows do not receive sufficient daylight to affect their circadian system [11] and therefore look for additional daylight and social interactions. There are other health damages that could happen (even cancer) [11]. The colour of the light is also important [5]. The luminous flicker of fluorescent lamps, which can be reduced or eliminated by replacing magnetic ballasts with digital ballasts, has shown to have affected visual performance, caused visual comfort and general stress [19].

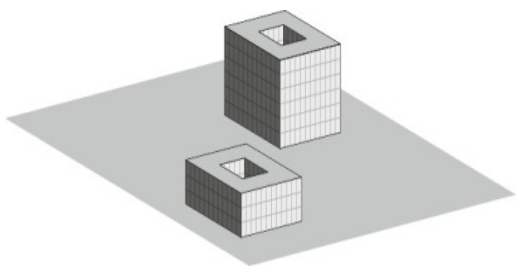

Figure 1: $\quad$ A perspective model of two atrium type buildings.

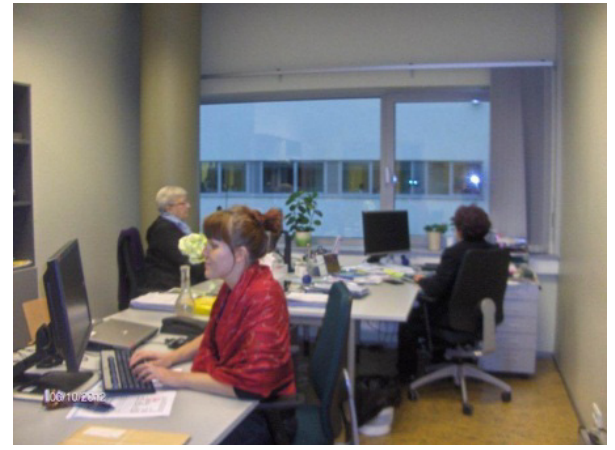

Figure 2: A workroom with the windows facing the atrium side is deficient of natural daylight. (Photo of an actual site where the measurements took place.)

Deficiency of natural daylight is especially common to workrooms facing an inner courtyard (such as an atrium) or another building close by. Visual ergonomics is unfortunately not considered as an important factor while designing a building on a drawing board. Therefore, any technical solution improving the conditions of natural lighting in such buildings should be thoroughly studied. A number of studies [17] exist that point out the important ergonomic characteristics of the artificial lighting system, such as: 1) a changing colour temperature, 2) a full spectrum of wavelengths, 3) ample light intensity and vertical lighting.

Biomedical studies conducted on workers having significantly different lighting conditions to those of natural daylight, may also have a disturbed wellbeing. The portion of the natural daylight indoors in wintertime at northern latitudes is no more than $10 \%$ of the lighting budget. Besides the visual ergonomics [11, 23], lighting has an important biological effect - entering the 
eyes and travelling through the optical nerves as an electrical signal, light reaches the brain which uses the energy produced by light to regulate and energize a variety of biological processes of the body (fig. 3). In general terms, light both directly and indirectly affects the human wellbeing.

Since the recent discovery of a new photoreceptor in the eye, the relevance of dynamic lighting has become clearer. Light and its characteristics are received by this newly discovered photoreceptor cells and processed by a separate nerve system, transmitted to the biological clock, which regulates the circadian (daily) and circannual (seasonal) rhythms of a range of bodily processes [24]. 24-hour daytime rhythms have both a physiological and behavioural impact on human health: wake/sleep cycles, alertness, core body temperature, performance patterns and production of hormones [23, 25].

Next to variations in the colour temperature a bright light itself is an important factor of natural daylight. Bright light is used in treating seasonal affective disorder (SAD). The reason for developing SAD is believed to be a deficiency or total lack of natural daylight, particularly in the winter period.

The symptoms of SAD include: depression, decreased interest in work or other activities, lack of energy, increased appetite with weight gain, carbohydrate and sugar cravings, increased need for sleep and excessive daytime sleepiness, social withdrawal, extreme afternoon slumps with decreased energy and concentration, also a decreased sex drive [15]. Bright light has been found to improve also social behaviour. People exposed to bright light with over $1000 \mathrm{~lx}$, benefited from less quarrelsome manners, more agreeable conduct and an overall better mood [21]. Bright light is a convenient method of treatment of nonseasonal depression and antidepressant medication $[15,17]$.

The mechanisms of the symptoms of SAD are still not fully understood by science. Some authors conclude that the blue component of the light is a determining factor in reducing SAD. Others have shown that also high intensity white light can be used for SAD treatment. There are also studies postulating that the colour of the light has no effect on SAD treatment as long as the light is intensive. Studies have even shown that intensive blue light can damage people's eyes [13]. Blue LEDs (light emitting diodes) while used daily in the treatment of SAD had a positive effect in relieving depression [14]. The same effect was not present for a control group that had the same therapy, but using dimmer red LEDs [14]. On the other hand, Thorn has shown in his study that SAD is linked to the cortisol awakening response (CAR) but there was no difference in cortisol levels in the course of the rest of the day in between the control groups [23]. It was concluded that during the reduced daylight period in winter time the cortisol response to waking up is attenuated in SAD persons [23]. Another study [19] concluded that at the same light intensities $\left(2.3 \times 10^{15}\right.$ photons $\left.\mathrm{si} \mathrm{cm}^{2}\right)$ white light had greater therapeutic effect than red or blue light.

Blue light is also considered affecting SAD by modulating emotion brain processing in healthy people [25]. Vandewalle et al. [25] carried out a study that demonstrated enhanced responses to auditory stimulus in the posterior hypothalamus in SAD patients; green light reduced these responses and blue or green light had no effect in the control group. Vandewalle's study succeeded in 
indicating posterior hypothalamus as the neurological substrate involved with $\mathrm{SAD}$, including a distinct response to light and affected emotional responses. The conclusion is that the light with its specific spectral characteristics has a direct effect on the emotional brain in SAD persons.

The importance of using natural daylight in lighting indoor workplaces is also emphasized by the new standard EVS 894:2008+A1:2010 [19], made available in 2011. The document stresses the human need for natural daylight. Earlier standards have regulated workplace lighting only from the aspect of artificial lighting [23].

The aim of this study was to test an indoor dynamic lighting model previously developed by the authors [21]. One of the intentions of this model was to compensate the deficiency of natural daylight in the winter period (especially common in the Nordic countries from October to February). Another aspect of the model - a focal interest of this study - was to match the colour temperature of the indoor lighting system to that of natural daylight. By doing so, the human organism will be better able to follow the circadian rhythms which signal the body, when it's time to be active and when it's time to rest.

\section{Material and method}

An experimental dynamic lighting panel was used to investigate the effect of dynamic colour temperature on the worker's well-being throughout the workday. The well-being was determined by a questionnaire, which also determined the overall symptoms and problems related to human visual capability and indoor lighting conditions. Additionally, a computerized test was used to measure the person's reaction speed changing from before to after the intervention.

The experimental lighting panel consisted of LED packets of various colours (blue, yellow, red) with total power consumption of up to $12 \mathrm{~W}$ (fig. 3). The LEDs switched on and off based on a preset timer relay. The combination of the different colour LEDs was aimed to imitate the colour temperature of natural daylight, while the Sun travels across the sky, from the morning's yellowish to the noon's bluish and forward to the evening's yellowish-reddish tone. The panel was fully automated and did not require any actions by the participator: the lights switched on at 8:00 (the beginning of the workday) and switched off at 20:00 (several hours after the end of the workday as some people tend to work past office hours). The lamp was equipped with a diffuser plate (size 430x295mm) that converted otherwise directional spotlights into a comfortable diffused light.

The experimental dynamic light panel was based on the measurements conducted by an earlier study mapping the conditions of natural daylight throughout the year [21]. The authors' previous study succeeded in creating a model for an indoor lighting system based on the actual daylight characteristics, such as illuminance level, ultraviolet level, spectrum and color temperature characteristics. As presented in fig. 4, the model prescribes an indoor lighting system to follow a constantly changing colour temperature curve dependent on the time of the day (colour temperature curve of the midsummer sun). Although the currently used experimental device is incapable of such precise tracking, a 


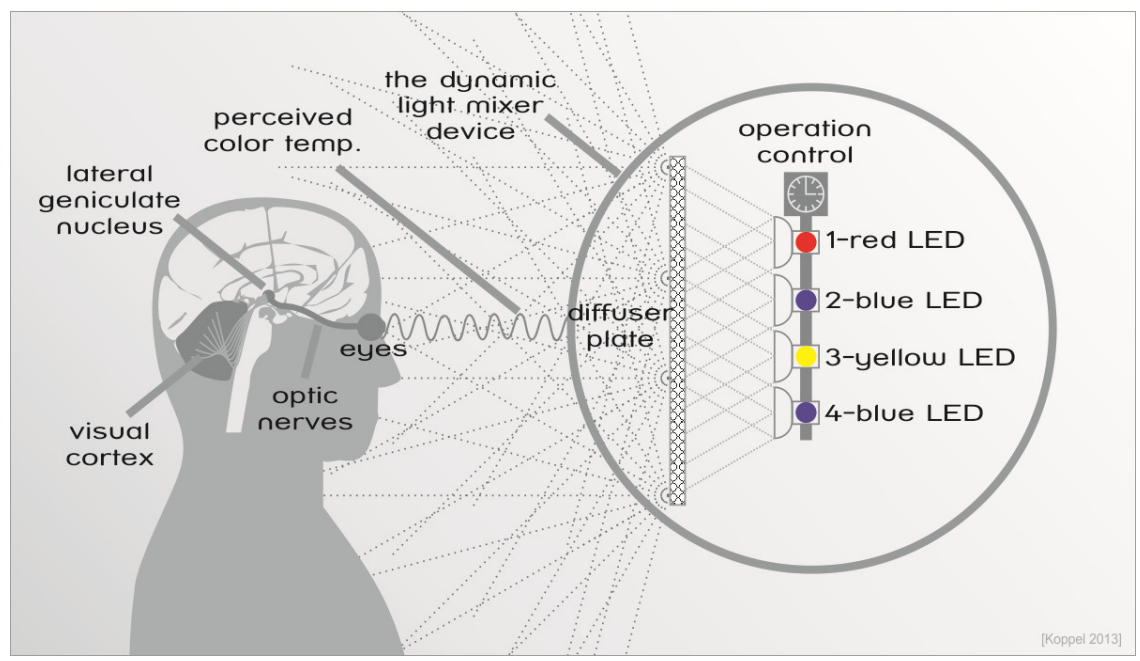

Figure 3: The conceptual model of the experimental dynamic light panel and the light's effective path into the human brain.

more crude approach was selected utilizing four packets of various colour LEDs. The basic prescription however remained the same: shifting the colour temperature from 2200-4300K throughout the day.

The authors consider the benefits of the dynamic lighting system tangible as that sort of light is already used as a pre-lunch countermeasure to circadian and sleep disruption in astronauts [24].

As the light perceived by the indoor worker consists both of artificial (ceiling and desktop) and natural lighting, the dynamic lighting panel was considered as a colour temperature additive to the present lighting conditions.

The light panel was placed vertically into the most common viewing area of the worker. As the office worker mostly looks at the computer screen, the panel was usually positioned in the vicinity of the computer monitor.

In a contrary to popular belief that selecting right colour temperature light bulbs helps to achieve the desired colour temperature settings in the room, the authors would like to point out that light will travel through and reflect from many surfaces, constantly having its colour temperature altered. Therefore it is necessary to measure and to intervene directly on the observer's line of sight.

In order to determine the perceived effects of the light panel, a questionnaire was filled out after the study. The main portion of the questionnaire was aimed to determine possible symptoms related to human visual performance and indoor lighting conditions. Also the satisfaction with the present lighting conditions at one's workplace was inquired of. In addition, the questionnaire mapped the general lighting conditions of the workroom. Also, the main personal characteristics and the time spent in the room were recorded by the questionnaire. 
The reaction speed test measured the person's finger reaction speed to an unforeseeable event on the computer screen. The overall test consisted of five consecutive events, the reaction time of which was averaged. The obtained reaction speed represents the person's eye-to-hand delay time and is expressed in milliseconds.

At each workplace the lighting conditions were metered by the researchers, recording minimal, maximum and average illuminance at the person's worktable. In two rooms the optical spectrometer was utilized to record the spectra and the colour temperature perceived by the worker under three different lighting scenarios: 1) only natural daylight (at noon), 2) indoor lighting in addition to previous, 3) dynamic lighting panel added to the previous. In the dynamic lighting panel mode the perceived colour temperature was metered in all lighting conditions: a) morning light, b) late morning light, c) noon light, d) afternoon light, e) evening light.

To perform the measurements, the following instruments were used:

1) optical spectrometer ASEQ Instruments LR-1; 2) laptop PC for real-time data recording and spectrometer control; 3) photometer Macam L203; 4) light meter Delta ohm hd2302.0; 5) UVA and UVB meter Waldmann 585 100; 6) UVC meter TIF3710. Indoor lighting measurements were conducted on the worktable and on the keyboard. The arithmetic mean, $\bar{E}$, was calculated.

\section{Results}

\subsection{Measurements}

The researchers used three sets of experimental dynamic lighting panels. The sets were distributed into workrooms facing the inner courtyard (the atrium) of the building. These workrooms were previously identified as having insufficient natural daylight conditions.

A total of 12 people participated in the experiment. In general, the intervention period lasted for three workdays after which the participator filled out the questionnaire. In conjunction to the questionnaire the researcher measured typical lighting conditions (without the light panel) of that workplace.

Illuminance levels of the participants' work desk were as also metered, the results demonstrated all the rooms to be adequately lit (mean $539 \mathrm{~lx}$ ) corresponding to a prevalent standard (legal requirement of $500 \mathrm{~lx}$. EN 124641:2002 "Light and lighting - Lighting of work places - Part 1: Indoor work places”).

Measurements of the illuminance level clearly showed that the indoor ceiling lighting system was sufficient in illuminating the work tasks in both atriumfacing workrooms and outside facing workrooms. Natural daylight conditions however differ drastically in atrium facing workrooms having an illuminance level of 49 lux and outside facing workrooms having the illuminance level of 421 lux on average. The measurements were conducted under heavy cloud coverage, typical to a winter day (so-called winter grey) conditions, around noon time. 


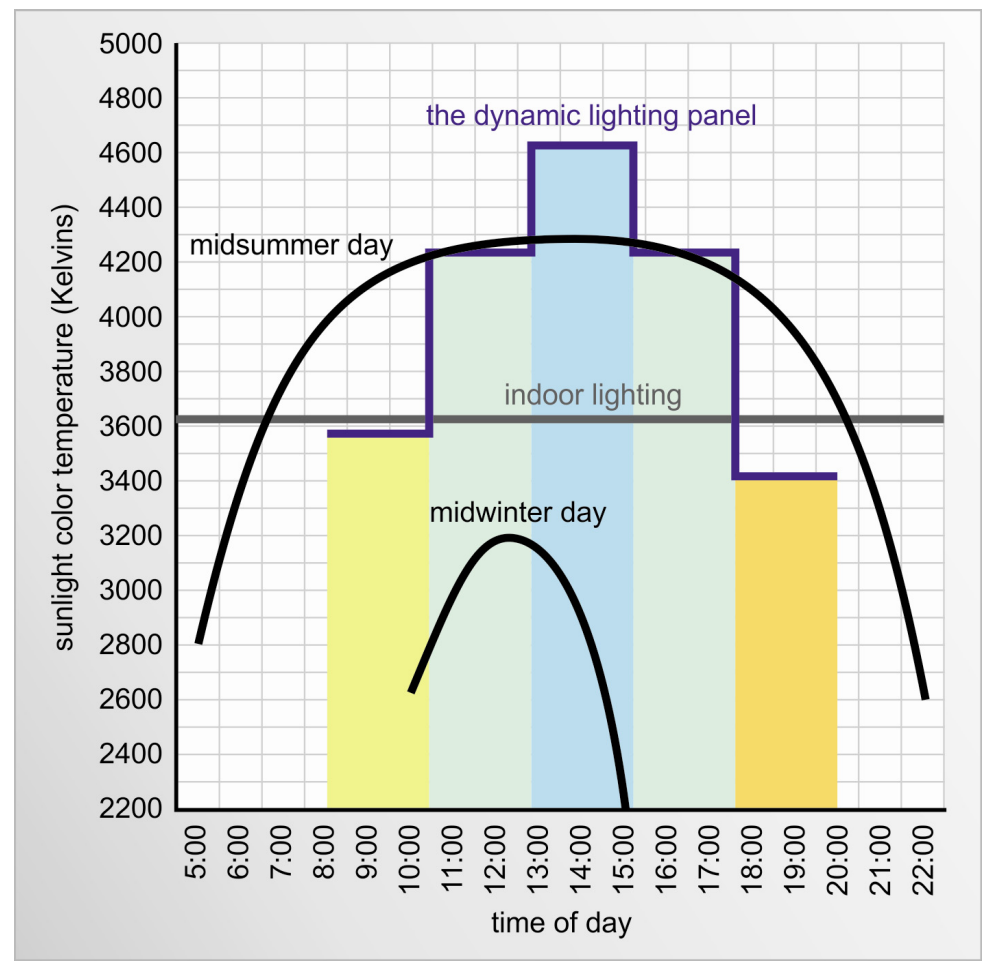

Figure 4: Indoor colour temperature measurements were done from the perspective of the human eye. The distance in between the viewer and the dynamic lighting panel was $0.6 \mathrm{~m}$. Outdoor measurements (midsummer's day and midwinter day) were conducted under direct sunlight conditions. Note the constant colour temperature of the indoor lighting.

From fig. 4, note the intensified characteristics of intervention colour temperature. This approach was selected since the worker is hardly likely to stare at the dynamic light panel all day but to keep his/her eyes on the computer, work desk, etc. Therefore, the perceived colour temperature consists of a mixture of light sources and in order for the dynamic lighting panel to have an effect it must have intensive extreme values. Why is the colour temperature to follow midsummer curve and not the midwinter curve - as research has pointed out the deficiency of natural daylight in the winter period (especially at the northern latitudes) is a good candidate for causing SAD and other adverse health effects, both physiological and cognitive. The authors postulated, that in order to overcome problems arising from deficient natural daylight in the winter, indoor lighting would be good to follow summertime colour temperature characteristics.

Although the dynamic lighting panel does not precisely follow the natural daylight dynamics still the colour temperature shift is achieved that corresponds 
to the overall trend of the natural daylight. One participant did complain about the blue light being over intensified.

\subsection{Feedback from the participators}

To obtain the feedback, questions were divided into four categories: 1) general adverse health symptoms related to visual functionality; 2) dissatisfaction with the current lighting conditions; 3) background information about the workplace, work regime and the person; 4) perceived effect from the intervention method.

Seven out of twelve people in the experiment reported having at least a moderate improvement from the intervention. Three people reported having some effect and replies from two people reported no effect. One person was even annoyed by the intensity of the blue light, feeling it to be too intensive. People reporting a positive effect also tended to point out the improved well-being and good mood in their comments.

Although the small sample size does not allow producing a rigid statistical analysis, Pearson's correlation indices were still calculated to implicate possible relationships between the reported beneficial effect and other parameters inquired of during the study. The highest correlation $(r=0.76)$ was linked to 1) the complaints about dry eyes and 2) the perceived beneficial effect from the dynamic lighting panel. It is no surprise that there was also a correlation of dry eye complaints in regard to the person's age $(r=0.81)$. This indicates that as the age accumulates, dry eyes are one of the symptoms that may accompany office workers. But also it might demonstrate that older persons and persons suffering from dry eyes benefit most from the intervention. Half of the participators expressed complaints about dry eyes. There are also some implications that if a person complained about 1) headaches or 2) anxiety/being irritated, he/she was also more likely to report improvement in general well-being $(r=0.62$ and $r=0.63)$ due to the intervention.

The data do tend to indicate that a positive effect succeeded the intervention. Deeper statistical analysis is however inconsiderable due to the small sample size and even the results presented should be taken with reservation as correlations inside the sample tend to be high in any study with a small sample.

Reaction speed tested before and after the intervention showed an average improvement of 10 per cent (from 338 to 299 milliseconds) across the sample. Although these results would indicate a remarkable change during the short intervention period, the authors would not overemphasize it, because of the methodological issues raised with the test setup: tests were conducted online and self administrated. Five of the subjects obtained more than 13 per cent improvement while the others had moderate or insignificant change (from 1 to 7 per cent). Improvements in reaction speed also seemed to have no relation to whether the person felt the intervention worked on him/her or not.

Out of ten symptoms listed, half of the participators complained about 1) tired eyes; 2) anxiety/being irritated and 3) dry eyes. All but one expressed high dissatisfaction with the current indoor lighting conditions, namely the lack of natural daylight. Expressed dissatisfaction about lighting conditions however does not seem to be connected to the amount of symptoms suffered or the 
perceived effect from the intervention. At the same time almost no complaints (but one) were made about the workplace being insufficiently illuminated. This shows that workrooms lit with standard fluorescent light tubes (with constant colour temperature and with large gaps in the spectrum) do provide adequate illuminance levels for performing tasks but do not fully cover the people's biological needs.

\subsection{Limitations}

Although the current experimental dynamic lighting panel is incapable of producing UV-light, the authors stress that a controlled UV exposure is a necessary component of a complete indoor dynamic lighting system. UV radiation is necessary for cell division - if a worker is lacking UV light, normal cell growth is disturbed, which can lead to cancer [18]. A workroom lack of sunlight also lacks UV-light. Despite popular belief that window glass blocks UV-light, this is only a half-truth, since the authors' measurement show that double glass windows allow an average 40 per cent of UV to pass (fig. 5). Therefore, workrooms facing an inner courtyard (an atrium) lack not only the dynamics of natural daylight but also UV-light - an integral part of daylight. Therefore it is yet not possible to determine which of natural daylight's

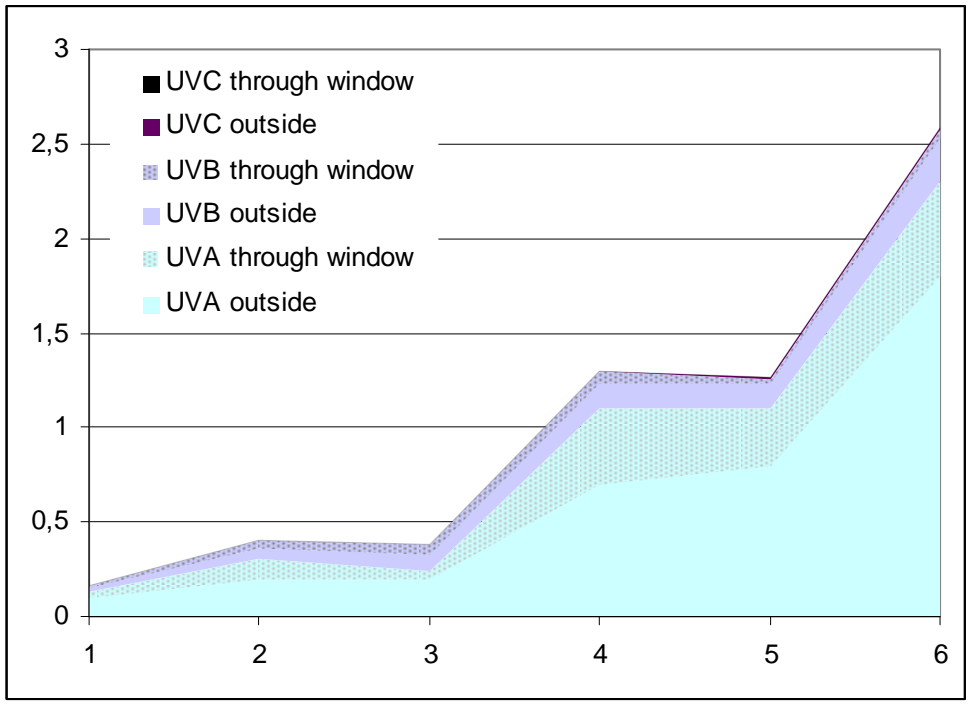

Figure 5: The coefficient of UV-light passing the window glass is 0.4 on average to UVA, UVB and UVC-light. Measured in six different occasions with different UV-levels (including winter- and summertime). Vertical axis: irradiance level $\left(\mathrm{mW} / \mathrm{cm}^{2}\right)$. Note the UVC levels being a thousand times smaller than UVA and therefore unnoticeable from the graph. (Source: author's measurements.) 
components (full spectrum, changing colour temperature or UV-light composition) acts most on the person's well-being; possibly all in some proportions.

\section{Conclusions and discussion}

The experimentally tested model for dynamic indoor lighting produced noticeable results in the perception and general well-being of the participators.

The main idea was to support the human day/night circadian rhythms. This is achieved by following the daylight colour temperature of the corresponding time of the day. Although the dynamic lighting panel can simulate some characteristics of the natural light, it can never achieve replacing the sunlight in its complexity.

Future research should focus on determining the precise effect of the light intervention. Current results only allow obtaining the general picture whether the intervention has a positive, a negative or no effect. The questioning methods should be further developed to understand why some of the participants were unaffected by the intervention device. Also, improvements should be made to the dynamic lighting panel itself, by having more colour temperature stages in the course of the day.

\section{Acknowledgements}

European Social Fund's Doctoral Studies and Internationalisation Programme DoRa supported this research. We also thank the project SF0140022s10 "Chemical Engineering Aspects in Environmental Risk Assessment” (Estonia) for the support.

\section{References}

[1] Bellia, L, Bisegna, F. and Spada, G., Lighting in indoor environments: visual and non-visual effects of light sources with different spectral power distribution. Building and Environment, 46, pp. 1984-1992, 2011.

[2] Boyce, P.R., Akashi, Y., Hunter, J. and Bollough, J., The impact of spectral power distribution on the performance of an achromatic visual task, Lighting Research and Technology, 35, pp. 141-161, 2003.

[3] Brainard, G.C., Sherry, D., Skwerer, R.G., Waxler, M., Kelly, K. and Rosenthal, N.E., Effects of different wavelengths in seasonal affective disorder, Journal of Affective Disorders, 20, pp. 209-216, 1990.

[4] Daurat, A., Aguirre, A., Foret, J., Connet, P., Keromes, A. and Benoit, O., Bright light affects alertness and performance rhythms during a 24-h constant routine, Physiology and Behaviour, 53, pp. 929-936, 1993.

[5] Deguchi, T. and Sato, M., The effect of color temperature of lighting sources on mental activity level. The Annals of Physiological Anthropology, 11(1), pp. 37-43, 1992. 
[6] DeKay, M., Daylighting and urban form: an urban fabric of light. Journal of Architectural and Planning Research, 27(1), pp. 33-56, 2010.

[7] Edwards, L. and Torcellini, P., A literature review of the effects of natural light on building occupants, National Renewable Energy Laboratory, US, Colorado, 2002.

[8] Even, C., Schröder, C.M., Friedman, S. and Rouillon, F., Effiacy of light therapy in nonseasonal depression: A systematic review, Journal of Affective Disorders, 108, pp. 11-23. 2008.

[9] EVS 894:2008+A1:2010. Daylight in dwellings and offices. Estonian Centre for Standardisation, 41 pp., 2010.

[10] EVS-EN 15251:2007. Indoor environmental inputs parameters for design and assessment of energy performance of building addressing indoor air quality, thermal environment, lighting and acoustics, Estonian Centre for Standardisation, 20pp., 2007.

[11] Figuero, M.G. and Rea, J.D., Does architectural lighting contribute to breast cancer? Journal of Carcinogenesis, 5, p. 20, 2006.

[12] Fucci, R.L., Gardner, J., Hanifin, J.P., Jassre, S., Byrne, B., Gerner, E., Rollag, M. and Brainard, G.C., Toward optimizing lighting as a countermeasure to sleep and circadian disruption in space flight, Acta Astronautica, 50, pp. 1017-1024,2005.

[13] Gagne, A-M., Levesque, F., Gagne, P. and Hebert, M., Impact of blue vs. red light on retinal response of patients with seasonal affective disorder and healthy controls, Progress in Neuro-Psychopharmacology \& Biological Psychiatry, 35, pp. 227-231, 2011.

[14] Glickmann, G., Byrne, B., Pineda, C., Hauck, W.W. and Brainard, G.C., Light Therapy for seasonal affective disorder with blue narrow-band light-emitting diodes (LEDs), Biol Psychiatry, 59, pp. 502-507, 2006.

[15] Holick, M.F. and Jenkins, M., The UV Advantage: new medical breakthroughs reveal powerful health benefits from sun exposure and tanning, $2^{\text {nd }}$ ed., Ener-Chi Wellness Press, 2011.

[16] Koppel, T., Dynamic Lighting System for Workplaces at Northern Latitudes. Scientific Journal of Riga Technical University, 15(3), pp. 3944, 2012.

[17] McColl, S.L. and Veitch, J., Full-spectrum fluorescent lighting: a review of its effects on physiology and health, Psychological Medicine, 31, pp. 949-964, 2001.

[18] Moritz, A., Heal yourself with sunlight, $2^{\text {nd }}$ ed, Ener-Chi Wellness Press, 2011.

[19] Nylén, P., Visual ergonomics. Book of abstracts. NES2012, eds A.-B. Antonsson, K. Vogel, G. M Hägg, KTH Royal Institute of Technology, School of Technology and Health, Sweden, p. 84, 2012.

[20] Reinhold, K. and Tint, P., "Lighting of Workplaces and Health Risks," Electronics and electrical engineering, 90, pp. 11-14, 2009.

[21] Rot, M., Moskowitz, D.S. and Young, S.N., Exposure to bright light is associated with positive social interaction and good mood over short 
periods: A naturalistic study in mildly seasonal people, Journal of Psychiatric Research,42, pp. 311-319, 2008.

[22] Song, K.D., Evaluating daylighting and heating designs of a top-glazed atrium space through physical scale model measurements and CFD analyses, Indoor and Built Environment,16, pp. 121-129, 2007.

[23] Thorn, L., Evans, P., Cannon, A., Hucklebridge, F., Evans, P. and Clow, A., Seasonal differences in the diurnal pattern of cortisolsecretion in healthy participants and those withself-assessed seasonal affective disorder, Psychoneuroendocrinology, 36, pp. 816-823, 2011.

[24] Van Bomml, W.J.M., Non-visual biological effect of lighting and the practical meaning for lighting your work, Applied Ergonomics, 37, pp. 461-466, 2006.

[25] Vandewalle, G., Hebert, M., Beaulieu, C., Richard, L., Daneault, V., Garon, M.-L., Leblanc, J., Grandjean, D., Maquet, P., Schwartz, S., Dumont, M., Doyon, J. and Carrie, J., Abnormal Hypothalamic Response to Light in Seasonal Affective Disorder, Biol. Psychiatry, 70, pp. 954961, 2011. 